\title{
Peripartum echocardiographic changes in women with hypertensive disorders
}

\section{of pregnancy}

Short title: Peripartum echocardiography and preeclampsia

\author{
V. Giorgione ${ }^{1,2}$, J. O’Driscoll 3,4 , C. M. Coutinho 2,5 , C. Di Fabrizio ${ }^{1}$, R. Sharma ${ }^{3}$, A. \\ Khalii ${ }^{1,2}$, B. Thilaganathan ${ }^{1,2}$
}

1. Molecular and Clinical Sciences Research Institute, St. George's University of London, London, UK

2. Department of Obstetrics and Gynaecology, St. George's University Hospitals NHS Foundation Trust, London, UK

3. Department of Cardiology, St George's University Hospitals NHS Foundation Trust, London, England.

4. School of Psychology and Life Sciences, Canterbury Christ Church University, Kent, England

5. Department of Gynecology and Obstetrics, Ribeirão Preto Medical School, University of São Paulo, Ribeirão Preto, SP, Brazil.

KEYWORDS: hypertensive disorders of pregnancy, preeclampsia, echocardiography, pregnancy, left ventricle remodelling

\section{CORRESPONDING AUTHOR: Professor B. Thilaganathan MD PhD FRCOG}

Fetal Medicine Unit, Department of Obstetrics and Gynaecology, St. George's University Hospitals NHS Foundation Trust. Blackshaw Road, London SW17 OQT, UK, E-mail: basky@pobox.com

This article has been accepted for publication and undergone full peer review but has not been through the copyediting, typesetting, pagination and proofreading process which may lead to differences between this version and the Version of Record. Please cite this article as doi: 


\section{CONTRIBUTION}

- What are the novel findings of this work?

Hypertensive disorders of pregnancies (HDP) are associated with abnormal left ventricle geometry and function; however, it is unknown if the hemodynamic responses related to volume redistribution following delivery have a significant impact on that. There was no significant difference in cardiac parameters in HDP women before and after delivery.

- What are the clinical implications of this work?

An echocardiographic evaluation in the peripartum period could be used to detect cardiac impairment in women affected by HDP and detect those who require closer surveillance in the postpartum period. This could be the first step in initiating a cardiovascular screening program after HDP that are acknowledged risk factors for cardiovascular diseases. 


\section{ABSTRACT}

Objectives: Women with hypertensive disorders of pregnancy (HDP) present with evidence of significant myocardial dysfunction on echocardiographic assessment at the time of diagnosis. Birth not only cures the syndrome of HDP, but is also associated with a reduction in cardiovascular $(\mathrm{CV})$ volume and resistance load in the mother due to the delivery of the fetoplacental unit. The impact of this physiological change on maternal myocardial function in women with HDP has not been systematically evaluated. The aim of this study is to compare echocardiographic findings immediately before and after childbirth in women with HDP.

Methods: In this prospective longitudinal study, 30 women with a diagnosis of HDP underwent two consecutive transthoracic echocardiography (TTE) examinations: the first prepartum and the second in the early postpartum period. Paired comparisons of these assessments were performed.

Results: Left ventricular (LV) concentric remodelling or hypertrophy were found in 21 (70\%) patients and there were no significant differences in cardiac morphology indices: LV mass index $\left(78.9 \pm 16.3 \mathrm{~g} / \mathrm{m}^{2}\right.$ vs $\left.77.9 \pm 15.4 \mathrm{~g} / \mathrm{m}^{2}, \mathrm{p}=0.611\right)$ and relative wall thickness $(0.45 \pm 0.1$ vs $0.44 \pm 0.1, p=0.453)$. LV diastolic function did not demonstrate any peripartum variation: left atrial volume $(52.40 \pm 15.3$ vs $50.97 \pm 15.6, p=0.433)$; lateral E' $(0.12 \pm 0.03$ vs $0.12 \pm 0.03, p=0.307)$ and $E / E '$ ratio $(7.88 \pm 2.19$ vs $7.91 \pm 1.74$, $p=0.934)$. Systolic function indices such as LV ejection fraction $(57.5 \pm 4.4 \%$ vs $56.4 \pm 2.1 \%, p=0.295)$ and global longitudinal strain $(-15.3 \pm 2.6 \%$ vs $-15.1 \pm 3.1 \%$, $\mathrm{p}=0.715$ ) also remained unchanged.

Conclusions: Maternal hemodynamic changes associated with birth did not significantly influence peripartum TTE indices in women with HDP. Suboptimal maternal echocardiographic findings in HDP are likely to be the consequence of chronic pregnancy CV load changes or pre-existing maternal CV impairment. Severity and persistence of myocardial dysfunction into the postpartum period may be related to the long-term maternal CV disease legacy of HDP. 


\section{INTRODUCTION}

Hypertensive disorders of pregnancy (HDP), including preeclampsia and gestational hypertension, are associated with substantial changes in maternal cardiac geometry and function during pregnancy and have been acknowledged as significant risk factors for cardiovascular disease ${ }^{1,2}$. In particular, women affected by preeclampsia show left ventricular (LV) diastolic dysfunction and remodelling/hypertrophy more frequently than women with a normotensive pregnancy ${ }^{3}$. The process of birth is accompanied by dramatic hemodynamic changes and alterations in circulating volume $e^{4}, 5$. In uncomplicated pregnancies, increased maternal stroke volume (SV) immediately after birth is thought to occur due to increased cardiac preload from autotransfusion of utero placental blood and reduction in the mechanical compression of the vena cava ${ }^{4}$. Assessment of hemodynamic variations using peripheral waveform devices have suggested similar improvement in hemodynamic parameters 2-3 days postpartum in HDP pregnancies ${ }^{6}$ and it has been proposed that maternal cardiovascular changes return to pre-pregnancy values within two weeks postpartum ${ }^{5}$.

Several studies demonstrate that maternal cardiac dysfunction is evident before the clinical onset of preeclampsia, is found several years after pregnancy and predisposes to the development of maternal cardiovascular morbidity long-term ${ }^{7-9}$. Peripartum echocardiographic assessment of LV diastolic function and geometry might be useful in women affected by HDP to identify markers of subsequent cardiovascular disease and associated adverse outcome ${ }^{10}, 11$. Ghossein-Doha et al. demonstrated that increased LV mass index at nine months postpartum was associated with the development of persistent chronic hypertension in formerly preeclamptic patients ${ }^{12}$. Similarly, other echocardiographic studies that investigated pregnancies complicated by HPD have been mostly conducted a few years after delivery, not in the immediate peripartum period ${ }^{8,9,12}$.

Therefore, it remains unclear whether maternal myocardial dysfunction in HDP is improved by the process of birth and whether this may have an influence on their ability to predict long-term maternal cardiovascular morbidity. The hypothesis is that giving birth could modify maternal cardiac changes that are usually observed in women affected by HDP and the primary outcome of the present study is to compare echocardiographic findings in women with HDP immediately before birth and in the early postpartum period. 


\section{METHODS}

\section{Study population}

Thirty women with a pregnancy complicated by HDP were recruited in the Maternity Department of St George's Hospital between February 2019 and August 2019. This was an observational longitudinal cohort study approved by the Local Ethics Committee (19/LO/0794) and all participants provided written informed consent. A sample size was not calculated because this is a pilot study of an ongoing larger adequately powered study. Medical history and obstetric data were collected from hospital records and HDP were diagnosed according to the criteria set by ISSHP ${ }^{13}$. Fetal growth restriction was defined as per the Delphi Consensus agreement ${ }^{14}$, while maternal height, weight and brachial blood pressure were obtained prior to hemodynamic assessment. Body mass index [BMI $\left.\left(\mathrm{kg} / \mathrm{m}^{2}\right)\right]$ was calculated by dividing body weight $(\mathrm{kg})$ by the squared height in meters $\left(\mathrm{m}^{2}\right)$. Body surface area [BSA $\left(\mathrm{m}^{2}\right)$ ] was measured using the following equation: $0.007184^{\star}$ height $(\mathrm{cm})^{0.725 *}$ weight $(\mathrm{kg})^{0.425}$. Systolic (SBP) and diastolic blood pressure (DBP) was obtained by an upper arm automatic blood pressure monitor (Microlife ${ }^{\circledR}$, Microlife AG Swiss Corporation, Switzerland) with the woman in a resting state using an appropriately sized cuff ${ }^{15}$. Mean arterial pressure (MAP) was calculated as $(2 * \mathrm{DBP}+\mathrm{SBP}) / 3$.

\section{Echocardiography}

All subjects underwent a transthoracic echocardiography (TTE) at rest in the left lateral decubitus position using a commercially available ultrasound system (GE Vivid ${ }^{\mathrm{TM}}$ E95; GE Healthcare, Horten, Norway). Two-dimensional and Doppler TTE was performed following the guidelines of the American Society of Echocardiography ${ }^{16,17}$. For each acquisition, three cardiac cycles of non-compressed data were stored in cine-loop format and analysed off-line by one investigator (VG) on a dedicated workstation (EchoPAC version 203, GE Healthcare, Horten, Norway). The investigator was blinded to the time of TTE. Using the two-dimensional parasternal long-axis view, left ventricular end-diastolic and end-systolic diameters (LVEDd and LVESd, respectively, in $\mathrm{mm}$ ), as well as thickness of the interventricular septum (IVST, in $\mathrm{mm}$ ) and of the posterior wall (PWT, in $\mathrm{mm}$ ) were measured. Left ventricular mass [LVM (g)] was calculated using the formula $0.8^{*}\left(1.04^{*}(\text { LVEDd+PWT+IVST })^{3}-\right.$ LVEDd $\left.^{3}\right)+0.6$ and indexed for BSA to obtain LVM index (LVMI). Relative wall thickness (RWT) was 
calculated as follows: RWT=2xPWd/LVEDd. Normal cardiac geometry, concentric remodelling, concentric hypertrophy and eccentric remodelling were defined according to guidelines ${ }^{17}$. The following diastolic indices were measured: i) peak E-wave velocity $(\mathrm{m} / \mathrm{s})$, peak A-wave velocity $(\mathrm{m} / \mathrm{s})$, their ratio $(E / A)$ and deceleration time $(\mathrm{ms})$ measured by pulsed wave Doppler; ii) lateral and septal $\mathrm{e}^{\prime}$ velocity $(\mathrm{m} / \mathrm{s})$ obtained by pulsed-wave tissue doppler imaging (TDI) at the lateral and septal mitral annulus; iii) E/e' where $e^{\prime}$ is the average of septal $e^{\prime}$ and lateral $e^{\prime} ;$ iv) left atrial maximum volume [LAV (mL)]; v) tricuspid regurgitation (TR) systolic jet velocity (m/sec) and vi) pulmonary vein (PV) S wave (m/s), PV D wave (m/s), PV A wave duration (ms) and PV S/D ratio. British Society of Echocardiography guidelines were applied to evaluate left ventricular diastolic function ${ }^{18}$. LV chamber radial systolic function was derived by measuring ejection fraction (EF) from Simpson's biplane method from apical 4chamber and 2-chamber views ${ }^{17}$. Moreover, cardiac mechanical function was assessed by using two-dimensional speckle-tracking strain imaging on 2-, 3- and 4chamber views with a frame rate of 60-90 frames/second ${ }^{19}$. Global longitudinal strain (GLS) quantification was performed using commercially available software (EchoPAC version 203, GE Healthcare, Horten, Norway). SV was calculated measuring the left ventricular outflow tract (LVOT) diameter, which was measured 3 to $10 \mathrm{~mm}$ from the aortic valve plane in midsystole with inner edge-to-inner edge methodology and the pulsed Doppler velocity time integral (VTI) in the 5-chamber view ${ }^{20}$. Cardiac output (CO) was obtained as the product of SV and heart rate (HR) derived from electrocardiographic (ECG) monitoring. Systemic vascular resistance (SVR) was calculated using MAP $\times 80 / C O$. Inter- and intra-observer reproducibility for echocardiographic measurements was assessed by offline analyses in 6 randomly selected subjects by two independent operators (VG and JOD).

\section{Statistical analysis}

Variables were assessed for normality by the Shapiro-Wilk test and by visualizing their histograms. Continuous data were expressed as mean \pm standard deviation (SD) or as median, interquartile range (IQR) according to the data distribution. Echocardiographic data were compared using paired t-test or Wilcoxon signed-rank test between before and after delivery. Statistical significance was deemed a priori as $p<0.05$. The analysis was performed using the statistical software packages SPSS 27.0 (SPSS Inc., Chicago, IL, USA). 


\section{RESULTS}

\section{Population description}

Twenty patients with preeclampsia and ten with gestational hypertension were included in the study (Table 1). TTE was performed at a median (IQR) of 4.5 (2-8) days before and 3.5 (2-6) days after birth. There were no significant differences in HR, blood pressure, SVR, SV and CO noted between pregnancy and immediate postpartum (Table 2). Maternal weight was significantly lower (mean weight lost $3.3 \pm 2.5 \mathrm{Kg}, \mathrm{p}<0.001$ ) after birth compared to prenatal assessment. Intra- and interobserver variability for echocardiographic variables assessed by TTE was excellent for the majority of parameters (Supplementary Table 1).

LV geometry, diastolic and systolic function

Giving birth did not affect LVM, LVMI, RWT, biplane LVEDV and ESV (Table 2). The same twenty-one (70\%) women demonstrated abnormal geometry (concentric remodelling or hypertrophy) both before and after birth (Table 2). Among them, the cardiac morphology of only one patient was classified as concentric hypertrophy and as concentric remodelling, respectively. The nine patients without any cardiac remodelling were affected by gestational hypertension in four cases and preeclampsia in five cases. Seventeen (56.7\%) women were identified with abnormal diastolic function (Grade I or grade II) both during pregnancy and immediately after delivery (Table 3) with no significant change in LAV, lateral E', E/E' and E/A ratio. There was a significant increase in septal E' $(0.09 \pm 0.02 \mathrm{~m} / \mathrm{s}$ vs $0.10 \pm 0.02 \mathrm{~m} / \mathrm{s}, p=0.035)$. However, biplane EF, average GLS, and twist and un-twist parameters were also unchanged before and after birth (Table 3, Figure 1). 


\section{DISCUSSION}

Despite considerable changes in maternal cardiovascular volume and resistance load induced at the time of birth, no substantial improvement in cardiac geometry and LV function indices were found between the TTE performed before and soon after giving birth in women with HDP. These findings suggest that maternal echocardiographic findings in HDP are likely to be the consequence of chronic pregnancy cardiovascular load changes and as such, need longer to resolve in the postpartum period. An alternative explanation is that maternal cardiac findings may not be a simple adaptative change to loading conditions in pregnancy, but point to pre-existing cardiac dysfunction with implications for long term maternal CV health.

\section{Interpretation of study findings and comparison with published literature}

Previous studies using unvalidated peripheral waveform devices in the peripartum period have shown that the high-output pregnancy circulation noted in the immediate postpartum resolves to pre-pregnancy levels within two weeks ${ }^{21,22}$. This increased volume load (preload) might be explained by the transfer of blood from the uterus into the systemic circulation and also due to improved venous return caused by decreased vena cava compression. Blood volume redistribution after HDP birth may be influenced by the characteristic antenatal findings of lower cardiac index, higher SVR and maladaptation to hemodynamic changes ${ }^{23}, 24$. Our data demonstrate that peripartum hemodynamic fluctuations result in only minimal changes in some indices of LV diastolic function and no alteration in LV geometry or systolic function.

Longitudinal studies investigating uncomplicated pregnancies reported that the greatest changes in cardiac morphology and biomarkers ( $\mathrm{N}$-terminal proB-type natriuretic peptide and high-sensitivity troponin I) occur within a week of childbirth and not during pregnancy ${ }^{25}$. This is consistent with the finding that a significant proportion of women with apparently normal pregnancies showed diastolic dysfunction and abnormal cardiac remodelling at term as signs of cardiovascular maladaptation to the volume-overloaded state ${ }^{26}$. Ambrozic et al. compared echocardiographic studies in 30 women with severe preeclampsia and 30 healthy controls who underwent TTE 1 day before, 1 and 4 days postdelivery. The findings of this study should be considered carefully as the comparison of cardiac geometry before delivery between these two 
groups was not in agreement with previous literature ${ }^{3}$. They showed that only normal pregnancies were able to respond in the immediate postpartum period by significantly increasing SV and transmitral E velocity compared to preeclamptic patients ${ }^{27}$. This difference in response could be explained by the fact that the maternal heart in hypertensive disorders is not capable of increasing SV with increased preload because of impaired LV function ${ }^{2,28}$.

\section{Clinical and Research Implications}

Several epidemiological studies have established that women affected by HDP in pregnancy are more prone to develop cardiovascular diseases later in life compared to women with uncomplicated pregnancies ${ }^{29}$. Notably, their cardiovascular risk increases very soon after birth, as demonstrated by a recent meta-analysis showing that the risk of developing chronic hypertension in women with a previous history of HDP was 6-fold higher than controls within the first 2 years following pregnancy ${ }^{30}$. Thus, the peripartum period represents a unique opportunity to assess the cardiovascular risk of HDP women and initiate a program of cardiovascular prevention and protection (Figure 2). In order to accomplish such a goal, identifying cardiac functional and structural abnormalities in the peripartum period in women with HDP might be vital considering their prognostic role in the general population to predict adverse outcome ${ }^{10}, 11$. For example, an increased LVMI in chronic hypertensive patients can be considered a prognostic factor for ischemic heart disease and heart failure as well as other established risk factors ${ }^{10}$.

A single-centre feasibility randomized controlled trial evaluated the effect of enalapril in the improvement of postnatal cardiovascular function in women affected by HDP who were recruited and studied by TTE in the immediate postpartum. Women treated with enalapril had improved diastolic function and LV remodelling at 6 months postpartum compared with placebo ${ }^{31}$. These cardiac improvements after targeted treatment have the potential to reduce long-term cardiovascular disease risk of women with a history of HDP. The data of the current study demonstrates that women affected by HDP can be screened using TTE for cardiac dysfunction either in pregnancy or in the immediate postpartum. Although further research on the potential use of TTE in the stratification of cardiovascular risk in women affected by HDP are needed, our 
findings will help to design a cardiovascular screening strategy able to identify those women who are at an increased risk of developing cardiovascular diseases. In addition, future prospective peripartum studies of HDP pregnancies that will include longer postpartum follow-ups may further help elucidate the hypotheses of preexitance of cardiovascular disease in pathological pregnancies and its link to longterm maternal cardiovascular health.

\section{Strengths and limitations}

This is a prospective study conducted in a single tertiary centre where all women were managed according to the local protocol and underwent an TTE that was analysed by the same operator (VG) that was blinded to the time where TTE was performed. Moreover, the prospective study design with a sufficient number of subjects, paired comparison and an application of novel echocardiographic modalities such as tissue Doppler imaging and speckle tracking echocardiography could be regarded as strengths of this study. A control group was not necessary because of the paired observations (before and after delivery) for each patient affected by HDP. The main limitation of the current study is related to the fact that multiple comparisons might have increased the risk of type I error for some of TTE indexes. Moreover, data on total blood volume assessed directly by invasive methods or by indirect methods were not available.

\section{Conclusion}

This study has demonstrated that maternal cardiac morphology and function in women affected by HDP does not improve in the postpartum period despite maternal physiological $\mathrm{CV}$ changes associated with birth. These findings suggest that maternal echocardiographic findings in HDP are likely to be the consequence of chronic pregnancy cardiovascular load changes and/or point to pre-existing cardiac dysfunction with implications for long-term maternal CV health. Maternal echocardiographic findings either immediately before birth or the early postpartum period may be used to tailor antihypertensive therapy or be used to assess long-term maternal cardiovascular health. 


\section{ACKNOWLEDGMENTS}

Veronica Giorgione and Carolina Di Fabrizio have received funding from the European Union's Horizon 2020 research and innovation programme under the Marie Skłodowska-Curie grant agreement No 765274 (iPLACENTA project).

Conrado Milani Coutinho's postdoctoral fellowship at St George's University Hospital was supported by funds from Fundação de Amparo à Pesquisa do Estado de São Paulo (FAPESP), process number 2019/01280-3

\section{CONFLICT OF INTEREST}

None 


\section{REFERENCE}

1. Parikh NI, Gonzalez JM, Anderson CAM, Judd SE, Rexrode KM, Hlatky MA, Gunderson EP, Stuart JJ, Vaidya D, American Heart Association Council on E, Prevention, Council on Arteriosclerosis T, Vascular B, Council on C, Stroke N, the Stroke C. Adverse Pregnancy Outcomes and Cardiovascular Disease Risk: Unique Opportunities for Cardiovascular Disease Prevention in Women: A Scientific Statement From the American Heart Association. Circulation 2021. DOI: 10.1161/CIR.0000000000000961. CIR0000000000000961.

2. Castleman JS, Ganapathy R, Taki F, Lip GY, Steeds RP, Kotecha D. Echocardiographic Structure and Function in Hypertensive Disorders of Pregnancy: A Systematic Review. Circ Cardiovasc Imaging 2016; 9.

3. De Haas S, Ghossein-Doha C, Geerts L, van Kuijk SMJ, van Drongelen J, Spaanderman MEA. Cardiac remodeling in normotensive pregnancy and in pregnancy complicated by hypertension: systematic review and meta-analysis. Ultrasound Obstet Gynecol 2017; 50: 683-696.

4. Ueland K, Hansen JM. Maternal cardiovascular dynamics. 3. Labor and delivery under local and caudal analgesia. Am J Obstet Gynecol 1969; 103: 8-18.

5. Robson SC, Hunter S, Moore M, Dunlop W. Haemodynamic changes during the puerperium: a Doppler and M-mode echocardiographic study. Br J Obstet Gynaecol 1987; 94: 1028-1039.

6. Lavie A, Ram M, Lev S, Blecher Y, Amikam U, Shulman Y, Avnon T, Weiner E, Many A. Maternal cardiovascular hemodynamics in normotensive versus preeclamptic pregnancies: a prospective longitudinal study using a noninvasive cardiac system (NICaS). BMC Pregnancy Childbirth 2018; 18: 229.

7. Garcia-Gonzalez C, Georgiopoulos G, Azim SA, Macaya F, Kametas N, Nihoyannopoulos P, Nicolaides KH, Charakida M. Maternal Cardiac Assessment at 35 to 37 Weeks Improves Prediction of Development of Preeclampsia. Hypertension 2020; 76: 514522.

8. Melchiorre K, Sutherland GR, Liberati M, Thilaganathan B. Preeclampsia is associated with persistent postpartum cardiovascular impairment. Hypertension 2011; 58: 709-715.

9. Reddy M, Wright L, Rolnik DL, Li W, Mol BW, La Gerche A, da SilvaCosta F, Wallace EM, Palmer K. Evaluation of Cardiac Function in Women With a History of Preeclampsia: A Systematic Review and Meta-Analysis. J Am Heart Assoc 2019; 8: e013545.

10. Modin D, Biering-Sorensen SR, Mogelvang R, Landler N, Jensen JS, Biering-Sorensen T. Prognostic Value of Echocardiography in Hypertensive Versus Nonhypertensive Participants From the General Population. Hypertension 2018; 71: 742-751.

11. Halley CM, Houghtaling PL, Khalil MK, Thomas JD, Jaber WA. Mortality rate in patients with diastolic dysfunction and normal systolic function. Arch Intern Med 2011; 171: 10821087.

12. Ghossein-Doha C, Peeters L, van Heijster S, van Kuijk S, Spaan J, Delhaas T, Spaanderman M. Hypertension after preeclampsia is preceded by changes in cardiac structure and function. Hypertension 2013; 62: 382-390.

13. Brown MA, Magee LA, Kenny LC, Karumanchi SA, McCarthy FP, Saito S, Hall DR, Warren CE, Adoyi G, Ishaku S, International Society for the Study of Hypertension in P. Hypertensive Disorders of Pregnancy: ISSHP Classification, Diagnosis, and Management Recommendations for International Practice. Hypertension 2018; 72: 24-43. 
14. Gordijn SJ, Beune IM, Thilaganathan B, Papageorghiou A, Baschat AA, Baker PN, Silver RM, Wynia K, Ganzevoort W. Consensus definition of fetal growth restriction: a Delphi procedure. Ultrasound Obstet Gynecol 2016; 48: 333-339.

15. Whelton PK, Carey RM, Aronow WS, Casey DE, Jr., Collins KJ, Dennison Himmelfarb C, DePalma SM, Gidding S, Jamerson KA, Jones DW, MacLaughlin EJ, Muntner P, Ovbiagele B, Smith SC, Jr., Spencer CC, Stafford RS, Taler SJ, Thomas RJ, Williams KA, Sr., Williamson JD, Wright JT, Jr. 2017 ACC/AHA/AAPA/ABC/ACPM/AGS/APhA/ASH/ASPC/NMA/PCNA Guideline for the Prevention, Detection, Evaluation, and Management of High Blood Pressure in Adults: A Report of the American College of Cardiology/American Heart Association Task Force on Clinical Practice Guidelines. J Am Coll Cardiol 2018; 71: e127-e248.

16. Mitchell C, Rahko PS, Blauwet LA, Canaday B, Finstuen JA, Foster MC, Horton K, Ogunyankin KO, Palma RA, Velazquez EJ. Guidelines for Performing a Comprehensive Transthoracic Echocardiographic Examination in Adults: Recommendations from the American Society of Echocardiography. J Am Soc Echocardiogr 2019; 32: 1-64.

17. Lang RM, Badano LP, Mor-Avi V, Afilalo J, Armstrong A, Ernande L, Flachskampf FA, Foster E, Goldstein SA, Kuznetsova T, Lancellotti P, Muraru D, Picard MH, Rietzschel ER, Rudski L, Spencer KT, Tsang W, Voigt JU. Recommendations for cardiac chamber quantification by echocardiography in adults: an update from the American Society of Echocardiography and the European Association of Cardiovascular Imaging. Eur Heart J Cardiovasc Imaging 2015; 16: $233-270$.

18. Mathew T SR, Jones R. A Guideline Protocol for the Echocardiographic assessment of Diastolic Dysfunction: British Society of Echocardiography. 2013.

19. Amzulescu MS, De Craene $M$, Langet $H$, Pasquet $A$, Vancraeynest $D$, Pouleur AC, Vanoverschelde JL, Gerber BL. Myocardial strain imaging: review of general principles, validation, and sources of discrepancies. Eur Heart J Cardiovasc Imaging 2019; 20: 605-619.

20. Bijl RC, Valensise H, Novelli GP, Vasapollo B, Wilkinson I, Thilaganathan B, Stohr EJ, Lees C, van der Marel CD, Cornette JMJ, International Working Group on Maternal H. Methods and considerations concerning cardiac output measurement in pregnant women: recommendations of the International Working Group on Maternal Hemodynamics. Ultrasound Obstet Gynecol 2019; 54: 35-50.

21. Ram M, Lavie A, Lev S, Blecher Y, Amikam U, Shulman Y, Avnon T, Weiner E, Many A. Cardiac hemodynamics before, during and after elective cesarean section under spinal anesthesia in low-risk women. J Perinatol 2017; 37: 793-799.

22. Hunter S, Robson SC. Adaptation of the maternal heart in pregnancy. Br Heart J 1992; 68: 540-543.

23. Dyer RA, Piercy JL, Reed AR, Lombard CJ, Schoeman LK, James MF. Hemodynamic changes associated with spinal anesthesia for cesarean delivery in severe preeclampsia. Anesthesiology 2008; 108: 802-811.

24. Kalafat E, Perry H, Bowe S, Thilaganathan B, Khalil A. Prognostic Value of Maternal Cardiovascular Hemodynamics in Women With Gestational Hypertension and Chronic Hypertension in Pregnancy. Hypertension 2020; 76: 506-513.

25. Umazume T, Yamada T, Yamada S, Ishikawa S, Furuta I, Iwano H, Murai D, Hayashi T, Okada K, Morikawa M, Yamada T, Ono K, Tsutsui H, Minakami H. Morphofunctional cardiac changes in pregnant women: associations with biomarkers. Open Heart 2018; 5: e000850.

26. Melchiorre K, Sharma R, Khalil A, Thilaganathan B. Maternal Cardiovascular Function in Normal Pregnancy: Evidence of Maladaptation to Chronic Volume Overload. Hypertension 2016; 67: 754-762. 
27. Ambrozic J, Lucovnik M, Prokselj K, Toplisek J, Cvijic M. Dynamic changes in cardiac function before and early postdelivery in women with severe preeclampsia. J Hypertens 2020; 38: 1367-1374.

28. Schotola H, Sossalla ST, Renner A, Gummert J, Danner BC, Schott P, Toischer K. The contractile adaption to preload depends on the amount of afterload. ESC Heart Fail 2017; 4: 468-478.

29. Melchiorre K, Thilaganathan B, Giorgione V, Ridder A, Memmo A, Khalil A. Hypertensive Disorders of Pregnancy and Future Cardiovascular Health. Front Cardiovasc Med 2020; 7: 59.

30. Giorgione V, Ridder A, Kalafat E, Khalil A, Thilaganathan B. Incidence of postpartum hypertension within 2 years of a pregnancy complicated by pre-eclampsia: a systematic review and meta-analysis. BJOG 2021; 128: 495-503.

31. Ormesher L, Higson S, Luckie M, Roberts SA, Glossop H, Trafford A, Cottrell E, Johnstone ED, Myers JE. Postnatal Enalapril to Improve Cardiovascular Function Following Preterm Preeclampsia (PICk-UP):: A Randomized Double-Blind Placebo-Controlled Feasibility Trial. Hypertension 2020; 76: 1828-1837. 


\section{FIGURE LEGEND}

Figure 1. Layer-specific longitudinal strain analysis by two-dimensional speckle tracking echocardiography for evaluating left ventricular systolic function in a woman affected by preeclampsia before delivery (upper three panes) and in the immediate postpartum (lower three panes).

Figure 2. Peripartum echocardiography in women affected by hypertensive disorders of pregnancy (HDP) as potential screening for cardiovascular diseases.

HDP hypertensive disorders of pregnancy, CVD cardiovascular diseases, LV left ventricle. Created with BioRender.com 
Table 1. Maternal demographics and obstetric characteristics $(n=30)$

\begin{tabular}{|c|c|}
\hline Maternal age (years) & $33.5 \pm 6.7$ \\
\hline Pre-gestational BMI & $26.8 \pm 5.3$ \\
\hline \multicolumn{2}{|l|}{ Ethnicity } \\
\hline Caucasian & $17(56.7)$ \\
\hline Afro-Caribbean & $8(26.7)$ \\
\hline Asian & $3(10.0)$ \\
\hline Nulliparity & $18(60)$ \\
\hline Chronic hypertension & $5(16.7)$ \\
\hline Pre-gestational diabetes & $1(3.3)$ \\
\hline Booking MAP (mmHg) & $94.1 \pm 6.4$ \\
\hline \multicolumn{2}{|l|}{ Diagnosis } \\
\hline Gestational hypertension & $10(33.3)$ \\
\hline Preeclampsia & $20(66.7)$ \\
\hline Fetal growth restriction & $7(23.3)$ \\
\hline Gestation at birth (weeks) & $35.9 \pm 4.0$ \\
\hline \multicolumn{2}{|l|}{ Preterm birth } \\
\hline$<37$ weeks & $15(50)$ \\
\hline$<34$ weeks & $9(30)$ \\
\hline \multicolumn{2}{|l|}{ Mode of delivery } \\
\hline Vaginal & $10(33.3)$ \\
\hline Caesarean & $20(66.7)$ \\
\hline Total blood loss at birth (mL) & $395(300-600)$ \\
\hline
\end{tabular}


Table 2. Maternal characteristics, hemodynamic and left ventricle geometry before and after delivery. Data are reported as n (\%), median (IQR) or mean (SD).

\begin{tabular}{|c|c|c|c|}
\hline Day difference, days & $4.5(2-8)$ & $3.5(2-6)$ & 0.310 \\
\hline Weight, Kg & $82.2 \pm 16.4$ & $78 \pm 16.6$ & $<0.001$ \\
\hline $\mathrm{SBP}, \mathrm{mmHg}$ & $135(128-144)$ & $134(129-143)$ & 0.742 \\
\hline $\mathrm{DBP}, \mathrm{mmHg}$ & $88(84-95)$ & $88(84-90)$ & 0.244 \\
\hline Patients on anti-hypertensive & $23(76.7)$ & $24(80)$ & 0.754 \\
\hline Stroke volume, $\mathrm{mL}$ & $64.0 \pm 10.6$ & $66.9 \pm 11.6$ & 0.253 \\
\hline Heart rate, bpm & $79.9 \pm 10.1$ & $80.0 \pm 8.7$ & 0.899 \\
\hline Cardiac output, L/min & $5.1 \pm 1.1$ & $5.4 \pm 1.2$ & 0.362 \\
\hline $\begin{array}{l}\text { Systemic vascular resistance, } \\
\text { dyne } / \mathrm{s} / \mathrm{cm}^{5}\end{array}$ & $\begin{array}{l}1571.43 \\
(1372.0-1809.8)\end{array}$ & $\begin{array}{l}1514.5 \\
(1255.4-1800)\end{array}$ & 0.139 \\
\hline \multicolumn{4}{|l|}{ Cardiac geometry } \\
\hline LVIDd (cm) & $4.40 \pm 0.61$ & $4.43 \pm 0.46$ & 0.661 \\
\hline LVIDs (cm) & $2.64 \pm 0.25$ & $2.61 \pm 0.46$ & 0.786 \\
\hline LV mass $(\mathrm{g})$ & $144.91 \pm 32.98$ & $144.72 \pm 33.41$ & 0.958 \\
\hline RWT & $0.45 \pm 0.09$ & $0.44 \pm 0.07$ & 0.453 \\
\hline Abnormal geometry & $21(70)$ & $21(70)$ & - \\
\hline Concentric Remodelling & 18 & 17 & \\
\hline Concentric Hypertrophy & 3 & 4 & \\
\hline Biplane EDV (ml) & $117.40 \pm 27.07$ & $120.82 \pm 26.34$ & 0.427 \\
\hline Biplane ESV (ml) & $50.17 \pm 12.83$ & $52.88 \pm 12.94$ & 0.194 \\
\hline
\end{tabular}

SBP systolic blood pressure, DBP diastolic blood pressure, LVIDd left ventricular internal diameter at end diastole, LVIDs left ventricular internal diameter at end systole, LV left ventricle, RWT relative wall thickness, EDV end-diastole volume, ESV end-systole volume. 
Table 3. Left ventricle diastolic and systolic function assessed by maternal echocardiography before and after delivery. Data are reported as n (\%), median (IQR) or mean (SD).

\begin{tabular}{|c|c|c|c|}
\hline \multicolumn{4}{|l|}{ Diastolic function } \\
\hline LA volume (ml) & $52.40 \pm 15.28$ & $50.97 \pm 15.56$ & 0.433 \\
\hline$M V E / A$ & $1.27 \pm 0.38$ & $1.22 \pm 0.27$ & 0.326 \\
\hline MV Deceleration time (ms) & $161.67 \pm 52.43$ & $157.53 \pm 37.54$ & 0.575 \\
\hline PV S/D & $1.26 \pm 0.26$ & $1.22 \pm 0.25$ & 0.499 \\
\hline Lateral E' (m/s) & $0.12 \pm 0.03$ & $0.12 \pm 0.03$ & 0.307 \\
\hline Septal E' (m/s) & $0.09 \pm 0.02$ & $0.10 \pm 0.02$ & 0.035 \\
\hline E/E' & $7.88 \pm 2.19$ & $7.91 \pm 1.74$ & 0.934 \\
\hline TR Vmax (m/s) & $2.10 \pm 0.46$ & $2.16 \pm 0.34$ & 0.487 \\
\hline Diastolic dysfunction & $17(56.7)$ & $17(56.7)$ & - \\
\hline Grade I & 3 & 5 & \\
\hline Grade II & 14 & 12 & \\
\hline \multicolumn{4}{|l|}{ Systolic function } \\
\hline Biplane EF (\%) & $57.52 \pm 3.42$ & $56.4 \pm 2.12$ & 0.295 \\
\hline Average GLS (\%) & $-15.31 \pm 2.64$ & $-15.13 \pm 3.10$ & 0.582 \\
\hline Apical Rotation (deg) & $9.82 \pm 5.62$ & $9.87 \pm 5.51$ & 0.998 \\
\hline Basal Rotation (deg) & $-6.80 \pm 5.67$ & $-8.02 \pm 4.06$ & 0.325 \\
\hline Twist (deg) & $17.05 \pm 6.41$ & $17.25 \pm 7.45$ & 0.979 \\
\hline Twist rate (deg/s) & $113.82 \pm 31.75$ & $122.16 \pm 53.94$ & 0.724 \\
\hline Untwist rate (deg/s) & $-124.98 \pm 46.03$ & $-134.53 \pm 41.96$ & 0.298 \\
\hline
\end{tabular}

LA left atrium, MV mitral valve, PV pulmonary vein, TR Vmax tricuspid regurgitation peak velocity, EF ejection fraction, GLS global longitudinal strain. 


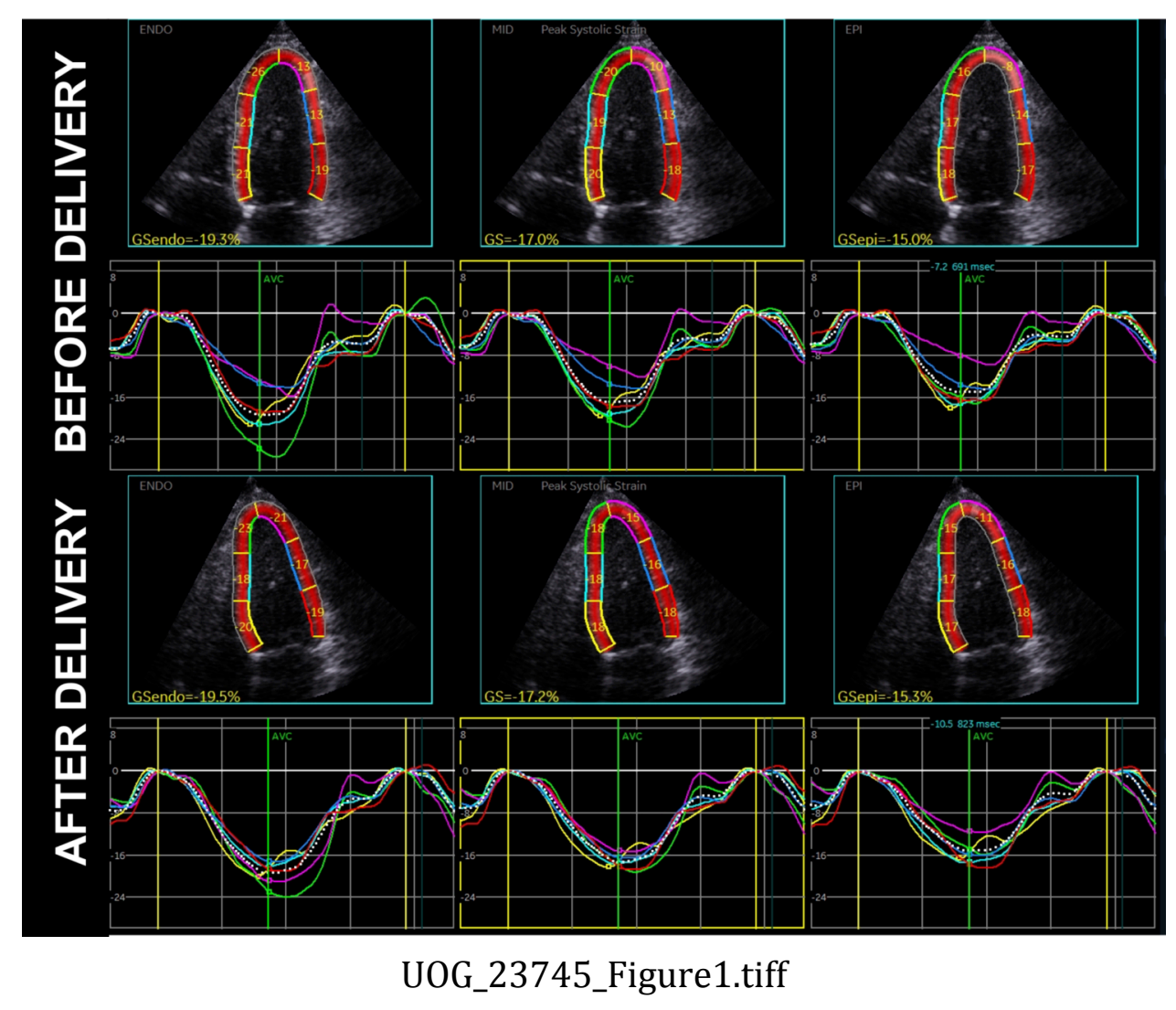




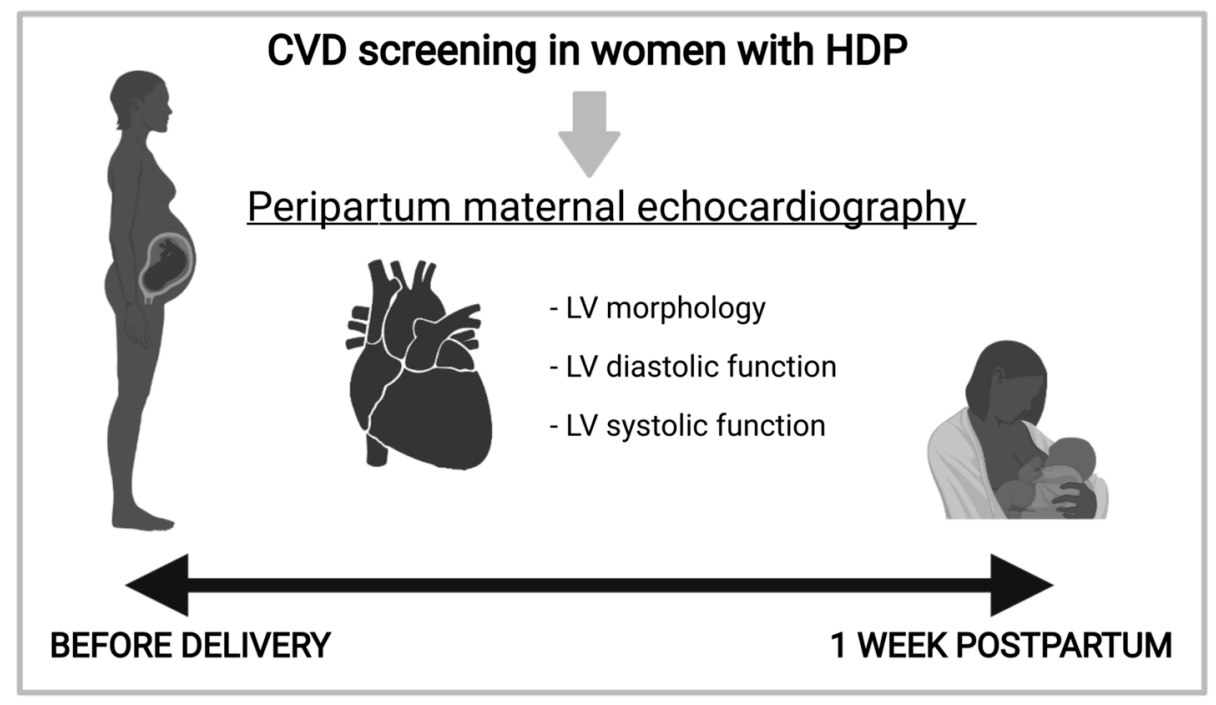

UOG_23745_Figure2.tiff 